\title{
REDES DE ATENÇÃO À SAÚDE: A PERCEPÇÃO DOS GESTORES MUNICIPAIS
}

\section{HEALTH CARE NETWORKS: THE PERCEPTION OF MUNICIPAL MANAGERS}

\section{REDES DE ATENCIÓN EN SALUD: PERCEPCIÓN DE LOS GESTORES MUNICIPALES}

\author{
Denise Finger ${ }^{1}$, Jeane Barros de Souza ${ }^{2}$,Valéria Silvana Faganello Madureira ${ }^{3}$, Daniela Savi \\ Geremia $^{4}$, Larissa Hermes Thomas Tombini ${ }^{5}$
}

Como citar esse artigo: Finger D, Souza JB, Madureira VSF, Geremia DS, Tombini LHT. Redes de atenção à saúde: a percepção dos gestores municipais. Rev Enferm Atenção Saúde [Internet]. 2021 [acesso em: ];10(1):e202105. Doi: https://doi.org/10.18554/reas.v10i1.3669

\section{RESUMO}

Objetivo: conhecer a percepção dos gestores municipais quanto ao papel da Secretaria Municipal de Saúde e da Secretaria Estadual de Saúde no processo de regionalização e organização das Redes de Atenção à Saúde. Método: estudo descritivo, exploratório, com abordagem qualitativa. A coleta de dados ocorreu no primeiro semestre de 2016, com 13 gestores municipais de saúde do estado de Santa Catarina. Os dados coletados foram analisados por meio da análise de conteúdo. Resultados: como potencialidades, evidenciou-se o suporte adequado da Secretaria Estadual de Saúde na educação permanente aos municípios e a participação do Conselho Municipal de Saúde na gestão e planejamento, bem como resultados positivos na assistência da Atenção Básica. Em relação às fragilidades, a principal citação foi referente ao repasse insuficiente de recursos financeiros. Conclusão: a cooperação entre municípios, a Comissão Intergestores Regional, as regiões e as Redes são importantes para sustentar a saúde nos pequenos municípios.

Descritores: Gestão em saúde; Governo local; Governo estadual; Sistema Único de Saúde; Assistência à saúde.

\footnotetext{
${ }^{1}$ Enfermeira. Especialista em Saúde da Família pelo programa de Residência Multiprofissional em Saúde da Família da Universidade Federal de Santa Catarina. Enfermeira na Secretaria Municipal de Saúde do município de Saudades-SC. deni.finger@hotmail.com. ORCID: https://orcid.org/0000-0001-8817-6745

${ }^{2}$ Enfermeira. Doutora em Ciências pela Universidade Federal de São Paulo. Pós-doutora em Enfermagem pela Universidade Federal de Santa Catarina. Professora Adjunta do Curso de Enfermagem da Universidade Federal da Fronteira Sul, Chapeco-SC, Brasil. jeanebarros18@gmail.com. https://orcid.org/0000-0002-0512-9765

${ }^{3}$ Enfermeira. Doutora em Enfermagem. Docente do curso de graduação em Enfermagem da Universidade Federal da Fronteira Sul - UFFS Campus Chapecó SC. valeria.madureira@uffs.edu.br. https://orcid.org/0000-0001-7990-3613

${ }^{4}$ Enfermeira. Doutora em Saúde Coletiva IMS/UERJ. Docente do curso de enfermagem da Universidade Federal da Fronteira Sul, Chapecó-SC. daniela.savi.geremia@gmail.com. https://orcid.org/0000-0003-2259-7429

5 Enfermeira. Doutora em saúde Coletiva. Professora Assistente da Universidade Federal da Fronteira Sul, Chapecó-SC.larissa.tombini@uffs.edu.br. https://orcid.org/0000-0002-6699-4955
} 


\begin{abstract}
Objective: to know the perception of municipal managers regarding the role of the Municipal Health Secretariat and the State Health Department in the process of regionalization and organization of Health Care Networks. Method: a descriptive, exploratory study with a qualitative approach. Data collection took place in the first half of 2016, with 13 municipal health managers from the state of Santa Catarina. The data collected were analyzed through content analysis. Results: as potentialities, it was evidenced the adequate support of the State Department of Health in the permanent education to the municipalities and the participation of the Municipal Health Council in the management and planning, as well as positive results in the assistance of Primary Care. Regarding the weaknesses, the main citation was referring to the insufficient transfer of financial resources. Conclusion: Cooperation between municipalities, the Regional Interagency Commission, regions and networks are important to sustain health in small municipalities
\end{abstract}

Descriptors: Health management; Local government; State government; Unified Health System, Delivery of health care.

\title{
RESUMEN
}

Objetivo: conocer la percepción de los gestores municipales sobre el papel de la Secretaría Municipal de Salud y de la Secretaría Estadual de Salud en el proceso de regionalización y organización de las Redes de Atención en Salud. Método: estudio descriptivo, exploratorio, con abordaje cualitativo. La recolección de datos se realizó el primer semestre de 2016, con 13 gestores municipales de salud del estado de Santa Catarina. Los datos recolectados fueron analizados por medio del análisis de contenido. Resultados: como potencialidades, se destacó el apoyo adecuado de la Secretaría Estadual de Salud en la educación continua de los municipios y la participación del Consejo Municipal de Salud en la gestión y planificación, así como también resultados positivos en la asistencia de la Atención Primaria. En cuanto a las debilidades, se mencionó principalmente la transferencia insuficiente de recursos financieros. Conclusión: la cooperación entre municipios, la Comisión Intergestores Regional, las regiones y las Redes son importantes para mantener la salud en los pequeños municipios

Descriptores: Gestión en salud; Gobierno local; Gobierno estadual; Sistema Único de Salud; Prestación de atención de salud; Atención de la salud.

\section{INTRODUÇÃO}

A gestão do Sistema Único de Saúde (SUS), é compartilhada pelas três esferas de governo: federal, estadual e municipal. Competências de cada esfera são apresentadas na Lei ${ }^{\circ} 8.080$ de 1990 que, além de dispor sobre as condições de promoção, proteção e recuperação da saúde, e da organização e funcionamento dos serviços, também apresenta as atribuições comuns e específicas de cada ente federado.
Aspectos relacionados à descentralização dos serviços, à regionalização, e às redes de atenção à saúde (RAS) são abordados na lei, determinando-os como responsabilidade dos governos federal, estadual e municipal. ${ }^{1}$

No processo histórico de construção e organização das ações em saúde no SUS, a descentralização se apresenta como princípio constitucional, conferindo a estados e municípios autonomia em relação à gestão da saúde. Neste contexto destacam- 
se as Normas Operacionais Básicas e do Pacto Pela Saúde, que conferiram aos municípios o papel central na implementação da política de saúde e na provisão dos serviços à população ${ }^{2}$, e aos gestores municipais o status de atores políticos estratégicos na definição e organização da política de saúde no âmbito local. ${ }^{3}$

No entanto, a maior autonomia municipal não exclui a responsabilidade de Estados e União, que permanecem com atribuições relacionadas à gestão e apoio, incluindo o repasse de recursos financeiros e de instrumentos políticos, administrativos, jurídicos e técnicos, para o efetivo funcionamento do sistema. ${ }^{4}$

As atribuições das três esferas de governo se estendem para a organização das RAS, que objetivam promover a integração de ações e serviços de saúde, a fim de proporcionar atenção contínua, integral, de qualidade, responsável e humanizada. ${ }^{5} \mathrm{~A}$ RAS é um conjunto de ações e serviços de saúde articulados em níveis de complexidade crescente, com o intuito de garantir a integralidade da assistência à saúde, sendo constituída por três elementos: população, estrutura operacional e modelo de atenção à saúde. ${ }^{5}$

Dado que a regionalização e a organização das RAS constituem princípios organizativos do SUS, fundamentais ao alcance da integralidade na atenção, e que sua operacionalização é sensível à percepção de gestores municipais quanto aos papeis das esferas envolvidas nos direcionamentos e decisões, justifica-se o presente estudo, que objetiva conhecer a percepção dos gestores municipais quanto ao papel da Secretaria Municipal de Saúde e da Secretaria Estadual de Saúde no processo de regionalização e organização das Redes de Atenção à Saúde.

\section{MÉTODOS}

O estudo consiste em uma pesquisa descritiva e exploratória, com abordagem qualitativa. A coleta de dados ocorreu no primeiro semestre de 2016, com os gestores dos municípios da região oeste do estado de Santa Catarina, pertencentes à Associação dos Municípios do Oeste de Santa Catarina, perfazendo o total de 27 municípios.

Participaram desse estudo os gestores dos municípios que aceitaram o convite por meio de contato telefônico e estiveram presentes na reunião da Comissão Intergestores Regional- CIR em dezembro de 2015. Dessa forma, contou-se com a participação de treze gestores municipais, que foram entrevistados após terem oficializado o aceite por meio da assinatura do Termo de Consentimento Livre e Esclarecido (TCLE). 
A coleta de dados foi realizada por meio de entrevistas, embasadas em um roteiro semiestruturado com sete perguntas, as quais foram gravadas em áudio e posteriormente transcritas. A pesquisa foi iniciada após a aprovação do Comitê de Ética em Pesquisa com Seres Humanos (parecer $\mathrm{n}^{\circ}$ 1.297.364), seguindo todas as preconizações da Resolução 466/2012 do Conselho Nacional de Saúde.

A organização e análise dos dados coletados foi baseada na análise de conteúdo. ${ }^{6}$ Assim, as seguintes etapas foram desenvolvidas: pré-análise; exploração do material ou codificação; tratamento dos resultados, inferência e interpretação.

Para garantir o sigilo dos participantes, esses foram identificados com nome de rios que formam a vertente do interior de Santa Catarina, composta pela bacia hidrográfica do Rio Paraná e do Rio Iguaçu, no intuito de realizar uma analogia entre a formação de rios e bacias hidrográficas com a construção das RAS na região do oeste catarinense.

\section{RESULTADOS E DISCUSSÕES}

A RAS é a forma atual de organização dos serviços de assistência à saúde no SUS, sendo que a União, o Distrito Federal e todos os estados e municípios são igualmente responsáveis, em todo o território nacional e em todos os níveis, pela sua implementação. ${ }^{7}$ Nesse sentido, os participantes do estudo relataram aspectos relacionados às atribuições do município na gestão do SUS e também abordaram sobre o trabalho desenvolvido pela Secretaria de Estado da Saúde (SES), destacando potencialidades e fragilidades.

\section{As atribuições e a atuação da SES}

No que tange ao papel da SES, os gestores citaram a coordenação, a organização dos serviços e o apoio financeiro:

\begin{abstract}
O papel da SES é de coordenação e organização dos serviços. E inclusive, de apoiar financeiramente porque os municípios não têm condições financeiras de estabelecer alguns serviços sozinhos [...] (Rio Peperi-Guaçu).
\end{abstract}

De acordo com o Conselho Nacional de Secretários de Saúde (CONASS) ${ }^{8}$, uma das funções da SES é promover a cooperação técnica entre os municípios, bem como realizar acompanhamento, avaliação e regulação dos serviços de saúde. O mesmo Conselho aborda a atuação do estado no cofinanciamento da Atenção Básica (AB), a partir de critérios e plano de investimentos.

Além do financiamento da $\mathrm{AB}$, os gestores discorreram sobre o papel da SES também no contexto da média e alta complexidade: 
Ela (SES) detém o poder de organizar o fluxo de pacientes de média e alta complexidade (Rio Canoinhas).

[...] o cofinanciamento é muito baixo e aquilo que é média complexidade, que deveria ser função do estado, não dá conta (Rio Negro).

Em relação à média e alta complexidade, o CONASS afirma a importante atribuição das SES, as quais atuam como principais agentes reguladores, a quem cabe coordenar e arbitrar as RAS, permitindo o acesso do usuário aos serviços necessários. $^{8}$

Ainda que o estado tenha papel de regulação de serviços, o incentivo à municipalização da gestão das RAS esbarra em dificuldades de acesso, especialmente na média e alta complexidade. Tal situação acarreta dependência externa, principalmente de municípios de menor porte e capacidade instalada, localizados em regiões remotas aos grandes centros urbanos, realidade no oeste catarinense. Neste sentido, o planejamento ascendente para a configuração das RAS se mostra fundamental no processo de tomada de decisão e de responsabilidades compartilhadas entre os municípios, devendo este ser mediado pelo estado. ${ }^{7}$

Aspectos relacionados ao não cumprimento de responsabilidades do estado, relacionadas ao financiamento, foram citadas:
Tem coisas que seriam responsabilidade do estado e a gente não 'tá' conseguindo, e vai acabar sempre aqui na nossa porta, nos municípios [...] (Rio Uruguai).

E eu acho que quando os municípios não atingem, é porque falta recursos e essa falta é aquela falta das obrigações do estado e da união [...] o estado consegue gastar só $4 \%$ do que arrecada, em saúde [...] (Rio Canoas).

Em relação às responsabilidades financeiras do estado, a Lei $n^{\circ} 141 / 2012$ estabelece os valores mínimos de responsabilidade de cada esfera do governo, no entanto, relata-se um grave déficit no investimento em saúde pelo estado. Essa ausência de suporte financeiro sobrecarrega e aumenta a responsabilidade dos municípios, os quais precisam arcar com os investimentos na $\mathrm{AB}$ e também em outras áreas.

Assim como a descentralização e a regionalização, o financiamento constitui recurso fundamental aos avanços e consolidação do SUS. Como tal, deve tripartite e equânime, de forma a garantir a implantação das RAS e a integralidade na atenção. ${ }^{9}$

A educação permanente foi citada como uma atribuição que está sendo desenvolvida adequadamente pela SES:

\footnotetext{
A Secretaria de Estado tem que ser a coordenadora de tudo, trazer a capacitação 'pra' nós gestores, os novos, os que já estão há mais tempo [...] (Rio Jangada).

Acho que o papel do estado é muito importante onde direciona os cursos, formação, recursos [...] (Rio Preto).
} 
De fato, um dos principais papeis da SES é apoiar e fornecer capacitações aos profissionais e gestores da rede de saúde. Isso também é citado pelo $\mathrm{CONASS}^{8}$, que reforça a importância de promover capacitações na $\mathrm{AB}$, principalmente voltadas à educação permanente e ao assessoramento aos municípios nas questões legais. Nesta perspectiva, os gestores entrevistados apontaram potencialidades da SES, como o suporte adequado na formação e apoio para os municípios:

A gente tem um suporte bem adequado da regional. [...] quanto às capacitações, informações de novas leis, isso a gente tem acesso (Rio Pelotas).

Percebe-se que, no que tange à oferta de cursos e apoio legal, a SES está em consonância com o CONASS, ofertando oportunidades e suporte adequado aos municípios através das unidades descentralizadas, as Secretarias de Desenvolvimento Regional, citadas pelo entrevistado como "regionais".

Com relação à organização dos serviços, os gestores abordaram a necessidade de descentralizá-los:

Falta uma ação efetiva nesse sentido, de descentralizar serviços, sair serviços da capital e aí o estado é fundamental para os recursos, para regionalizar [...] recursos são poucos, a gente sabe que 'vão ser' poucos sempre e muito menos ainda se a gente não fizer uma gestão eficiente (Rio Negro).
Percebe-se a necessidade de maior descentralização dos serviços de saúde, no entanto, os estados ainda enfrentam dificuldades relacionadas à fragilidade da articulação e coordenação dos sistemas estaduais e das RAS. ${ }^{10}$ Evidencia-se também que os gestores possuem conhecimento das dificuldades financeiras, mas ainda percebem a regionalização como uma estratégia para otimizar os recursos financeiros, realizando uma gestão eficiente.

Outro obstáculo referenciado foi a interferência político-partidária na gestão do SUS:

[...] dá a impressão de que tudo que eles fazem e falam é mais para fazer política do que para querer fazer saúde (Rio Canoas). Muitas vezes existem outros atores no meio dessa peça que vão lá dizer que o governo não precisa fazer um hospital aqui $e$ precisa bancar o hospital lá, precisa disso, precisa daquilo [...], sem fazer de fato o que seria mais necessário (Rio Negro).

Essas falas evidenciam que a saúde ainda é considerada um campo de atuação político-partidária. Essa interferência está presente nas decisões em nível estadual e também nos pequenos municípios, e foi apontada como obstáculo em outros estudos. ${ }^{11,12}$

Assim, é importante refletir sobre a democracia e a dificuldade de compreensão da saúde como um direito de cidadania e não moeda de troca de favores. No Brasil, a 
legitimidade democrática não está isenta da representação de interesses e o apoio da sociedade civil ao governo está amplamente influenciado por questões partidárias, mídia, redes sociais, grupos e movimentos que lutam por interesses particularizados, que podem ou não ser coletivos. ${ }^{13}$

O direcionamento de gestão e de recursos a partir de interesses partidários não cabe em um sistema de saúde como o SUS, cujos princípios valorizam a universalidade de acesso e de atenção. Entretanto, os municípios, especialmente os de pequeno porte, parecem sentir ainda as influências político-partidárias, as quais exercem pressão sobre gestores, legisladores, reguladores e profissionais da saúde, refletindo diretamente no atendimento à população. Nesse sentido, percebe-se como a elite hegemônica utiliza o poder para fins próprios, fazendo do povo a base de sua legitimação ${ }^{11}$, traduzindo-se na dominação de alguns atores políticos sobre os gestores e profissionais da saúde, bem como sobre o restante da população.

Os municípios são autônomos e as eventuais políticas estaduais só são viáveis sob uma lógica eminentemente cooperativa. Mas na ausência da relação hierárquica, a relação cooperativa passa a ser fortemente condicionada pelas relações políticopartidárias, pois inexiste na Federação brasileira, a dimensão estadual de controle dessas políticas. ${ }^{14}$

O processo de descentralização da gestão do SUS para os municípios contribuiu diretamente para a melhoria do acesso da população aos serviços de saúde ${ }^{10}$, no entanto, afastou os estados da regulação dos sistemas regionais, ficando abstrata a sua função, demonstrando que o exercício político ainda necessita ser aprimorado.

\section{As atribuições e a atuação das SMS}

Em relação ao papel das Secretaria Municipais de Saúde (SMS), os entrevistados evidenciaram aspectos como atendimento da $\mathrm{AB}$, organização da média e alta complexidade, necessidades da população, e responsabilidade financeira:

\footnotetext{
O papel da SMS é organizar o atendimento na $A B$ e os fluxos de média e alta complexidade, e auxiliar na estruturação nas redes de urgência e emergência (Rio Canoinhas).

Identificar as necessidades da população, na implantação dos serviços. Ajudar tanto financeiramente quanto na estrutura (Rio Peperi-Guaçu).
}

De fato, o município possui um papel primordial na regionalização e organização das RAS, pois é a esfera de gestão mais próxima da população. Tal posição the confere responsabilidade maior em relação à $\mathrm{AB}$, desenvolvida de forma descentralizada e capilarizada, sendo a 
porta de entrada preferencial do usuário na RAS. $^{15}$

Além de desenvolver $\mathrm{AB}$ de qualidade em cada município, é necessário ter objetivos em comum e cooperação entre diferentes municípios, a fim de formar uma rede regionalizada de atenção à saúde. Nesse aspecto, destacam-se alguns relatos:

\begin{abstract}
Eu acredito que é bem importante a participação de todos os municípios. É por isso que a gente tem as nossas reuniões, as reuniões da CIR, reunião do colegiado [...] (Rio Jacutinga).

O nosso papel é fazer parte, é colocar o município à disposição e colocar os serviços de saúde que a gente tem no nosso município para os demais, e também não se preocupar só com o nosso município, fazer com que o nosso município também participe das redes, e busque essa regionalização como forma de melhorar o atendimento, melhorar acesso e também melhorar custos desses atendimentos (Rio Jangada).
\end{abstract}

Por se tratar de condições semelhantes entre os municípios, a maioria de pequeno porte, é interessante perceber que os entrevistados abordaram a necessidade de trabalhar em redes regionalizadas, enfocando a cooperação entre municípios e a participação em colegiados e na CIR, que são importantes espaços de negociação e pactuação. Assim, percebe-se que a cooperação entre os municípios e a construção das RAS têm sido a forma de sustentar a saúde nos pequenos municípios da região oeste catarinense.
Ainda, considerando as atribuições destacadas, vale salientar a responsabilidade dos gestores municipais de saúde, juntamente com o Conselho Municipal de Saúde (CMS), pela condução político-administrativa do sistema. Entre essas responsabilidades pode-se destacar a articulação da SMS com o legislativo e o executivo municipal, bem como com demais organizações comunitárias. Essa articulação é essencial para a SMS planejar, programar, conduzir, controlar e avaliar o sistema municipal de saúde; negociar com o setor privado ou conveniado com o SUS; organizar o modelo de assistência à saúde no município e administrar recursos humanos, financeiros e materiais que compõem a estrutura do sistema municipal de saúde. ${ }^{4} \mathrm{O}$ papel do CMS na gestão municipal foi citado por alguns:

\section{[...] tem algumas particularidades que são municipais, então a gente senta e discute no CMS e através do plano, o que é prioridade (Rio Jacutinga). \\ Nós temos o Conselho e daí todas as decisões que tomamos a gente leva pro Conselho, senta com o Conselho, discute e vê a melhor maneira de planejar e de se trabalhar (Rio Uruguai).}

De acordo com os participantes, percebe-se que os municípios possuem CMS atuante na gestão do SUS. A gestão municipal de saúde não é realizada apenas pela figura do secretário de saúde, mas inclui a participação e o controle social, o 
que foi instituído legalmente em 1990, através da Lei $\mathrm{n}^{\circ} 8.142$.

Os gestores ainda relataram outras ações desenvolvidas em seus municípios, destacando a cobertura da Estratégia Saúde da Família (ESF) e a atuação da AB como algo favorável na região:

[...] os municípios da região, na parte de atenção básica, todos eles fazem muito bem, fazem atenção básica boa, de qualidade (Rio Canoinhas).

A gente tem cobertura de $100 \%$ de ESF, a gente tem uma boa cobertura de saúde bucal, os programas de saúde a gente tem implantado (Rio Negro).

De fato, os municípios devem responsabilizar-se pela $\mathrm{AB}$, já que constitui 'porta de entrada preferencial' do SUS e nível de atenção mais próximo dos usuários e de suas reais necessidades. Nesse sentido, os estados e municípios brasileiros são os principais atores da descentralização, pois estão mais próximos da população e conhecem a realidade, tornando-se, dessa forma, aptos a definir a dinâmica dos espaços e as ações que serão desenvolvidas nesse território ${ }^{16}$. Os municípios são protagonistas desse processo, lembrando que os estados devem cumprir sua função de articulação regional, prestando apoio aos municípios. ${ }^{17}$

É importante destacar que não apenas os gestores municipais, mas os profissionais que atuam na $\mathrm{AB}$, dentre eles o enfermeiro, precisam reconhecer $\mathrm{o}$ funcionamento e a importância das RAS para valorizar os sistemas de apoio e logístico e, assim, buscar garantir a integralidade do cuidado e estratégias de articulação entre os serviços, a fim de consolidar os princípios do SUS. ${ }^{18}$

Os participantes também referenciaram a sobrecarga dos municípios, que precisam destinar recursos para a atenção de alta e média complexidade, prejudicando a $\mathrm{AB}$ :

[...] o município está assumindo um fardo
bastante pesado e não só o nosso, mas a
gente vê a região toda, os municípios
acabam tirando recurso da AB para pôr na
média complexidade. Isso não deveria
acontecer. Como é que nós vamos melhorar
a qualidade da AB se nós tiramos recursos
para colocar na media complexidade? (Rio
Negro).
O processo de descentralização do SUS foi muito forte e, nele, quem mais ampliou os gastos com saúde foram os municípios. Assim, muitos municípios evoluíram em direção à universalidade do sistema, mas se encontram impotentes e incapazes de avançar solitariamente nas ações em saúde. ${ }^{17}$

Conforme os entrevistados, os municípios também sofrem com a falta de autonomia para decidir o destino dos recursos:

Eu acho que poderia vir mais [recursos] para o município gerir... Nós que estamos no município sabemos das necessidades [...] 
então acho que deveria vir para que eu pudesse decidir mais (Rio Pelotas).

É importante salientar que o repasse dos recursos federais, na oportunidade, ocorria por meio de seis blocos de financiamento: $\mathrm{AB}$, atenção de média e alta complexidade ambulatorial e hospitalar, vigilância em saúde, assistência farmacêutica, gestão do SUS e, investimentos na rede de serviço de saúde. ${ }^{19}$ Os relatos sinalizam as dificuldades do trabalho e reduzida autonomia na alocação dos recursos de acordo com as realidades locais.

Percebe-se que as principais dificuldades enfrentadas pelos gestores municipais se referem ao financiamento, tanto em relação à falta de autonomia, como à falta de apoio das demais esferas do governo, resultando em um efeito bola de neve, em que necessitam investir recursos próprios em ações de média e alta complexidade, prejudicando a $\mathrm{AB}$, o que pode desestabilizar as RAS.

A antiga demanda dos gestores municipais de saúde para extinção dos blocos foi aprovada pela Portaria $n^{\circ} 3.992$, de 28/12/2017 20 , e as transferências fundo a fundo passaram a ser consolidadas em custeio e investimento, o que permitiu que estados e municípios remanejassem recursos seguindo planejamento próprio.
A nova portaria representa maior flexibilidade operacional na utilização dos recursos. Todavia, há a necessidade de se definir quais serão os critérios de alocação dos recursos, pois essa medida poderá gerar distorções, retirando recursos necessários para a $\mathrm{AB}$ e as ações de vigilância em saúde, por exemplo, para realocá-los em média e alta complexidade.

\section{CONCLUSÃO}

Apesar das diretrizes de gestão compartilhada e acesso integral no âmbito do SUS, observam-se lacunas à concretização destes, especialmente evidenciadas por gestores de saúde de municípios de menor porte e capacidade instalada, que vivenciam diariamente dificuldades na tomada de decisões e no acesso em uma rede limitada de serviços, realidade no oeste catarinense e, demais regiões do Brasil.

Apesar da descentralização de serviços de saúde para a região oeste, persiste a concentração de serviços de média e alta complexidade na capital do estado, cujo deslocamento de usuários representa gastos adicionais às SMS.

Ainda, a interferência políticopartidária nas decisões atinge as gestões local e estadual, instigando reflexões sobre a saúde como direito constitucional versus instrumento de poder, já que comumente 
tornou-se objeto de autopromoção política, garantida ao usuário por meio do voto.

Apesar das fragilidades, avanços foram citados, como a participação do CMS na gestão e planejamento e, os resultados positivos na assistência da $\mathrm{AB}$, cuja cobertura da ESF é de $100 \%$ em muitos municípios estudados. Nesse cenário observa-se que a prioridade na região é o aprimoramento da atenção secundária.

Quanto às atribuições da SES, potencialidades como o suporte adequado na oferta de cursos de formação para os gestores, e o apoio à atualização sobre legislação e normativas vigentes, foram citados. No entanto, o financiamento em saúde é ponto nevrálgico. A insuficiência de recursos para atendimento da demanda, especialmente em média e alta complexidade, é destaque pelos gestores, assim como a falta de autonomia destes nas decisões no nível regional.

Apesar das dificuldades apontadas, percebe-se grande evolução na regionalização e descentralização da gestão do SUS. Desta forma, reafirmam-se a cooperação entre municípios, as CIR, as regiões de saúde e as RAS regionalizadas como alternativas para a sobrevivência dos pequenos municípios no oeste catarinense.

A não abrangência de todos os gestores municipais da região estudada constituiu uma limitação deste estudo, já que diferentes percepções podem não ter sido captadas e consideradas nos resultados apresentados. Neste sentido, sugere-se a continuidade de pesquisas e a permanente discussão desta temática, envolvendo os diretamente implicados nas decisões e gestão em saúde, para a qualificação da atenção e consolidação do SUS nos âmbitos locais e regionais.

\section{REFERÊNCIAS}

1. Presidência da República (Brasil). Lei $\mathrm{n}^{\circ}$ 8.080, de 19 de Setembro de 1990a. Dispõe sobre as condições para a promoção, proteção e recuperação da saúde, a organização e o funcionamento dos serviços correspondentes e dá outras providências [Internet]. DOU, Brasília, DF, 20 set 1990 [citado em 03 jul 2020]. Disponível em: http://www.planalto.gov.br/ccivil_03/l eis/18080.htm

2. Menicucci TMG, Costa LA, Machado JA. Pacto pela saúde: aproximações e colisões na arena federativa. Ciênc Saúde Colet. [Internet]. 2018 [citado em 12 ago 2020]; 23(1):29-40. doi: https://doi.org/10.1590/141381232018231.17902015

3. Ouverney ALM, Carvalho ALB, Machado NMS, Moreira MR, Ribeiro JM. Gestores municipais do sistema único de saúde: perfil e perspectivas para o ciclo de gestão 2017-2020. Saúde Debate [Internet]. 2019 [citado em 12 ago 2020]; 43(N Esp 7):75-91.

Disponível em: http://www.scielo.br/scielo.php?script= sci_arttext\&pid=S010311042019001200075\&lng=pt. doi: https://doi.org/10.1590/010311042019 s706 
4. Teixeira CF, Molesini JA. Gestão municipal do SUS: Atribuições e responsabilidades do gestor do sistema e dos gerentes de unidades de saúde. Rev Baiana Saúde Pública [Internet]. 2002 [citado em 15 jun 2016]; 26(1-2): 29-40. Disponível em: http://rbsp.sesab.ba.gov.br/index.php/r bsp/article/view/983

5. Presidência da República (Brasil). Decreto $\mathrm{n}^{\circ} 7.508$, de 28 de Junho de 2011. Regulamenta a Lei no ${ }^{\circ} .080$, de 19 de setembro de 1990, para dispor sobre a organização do Sistema Único de Saúde - SUS, o planejamento da saúde, a assistência à saúde e a articulação interfederativa, e dá outras providências [Internet]. DOU, Brasília, DF, 29 jun 2011 [citado em 03 jul 2020]. Disponível em: http://www.planalto.gov.br/ccivil_03/ ato20112014/2011/decreto/d7508.htm

6. Bardin NL. Análise de conteúdo. São Paulo; 2011.

7. Peiter CC, Santos JLG, Lanzoni GMM, Mello ALSF, Costa MFBNA, Andrade SR. Redes de atenção à saúde: tendências da produção de conhecimento no Brasil. Esc Anna Nery Rev Enferm. [Internet]. 2019 [citado em 12 ago 2020]; 23(1): e20180214. Disponível em: http://www.scielo.br/scielo.php?script $=$ sci_arttext\&pid=S141481452019000100801\&lng=en. doi: https://doi.org/10.1590/2177-9465ean-2018-0214

8. Conselho Nacional de Secretários de Saúde (Brasil). A gestão do SUS. Brasília, DF: CONASS; 2015. 133 p.

9. Moreira LMC, Ferré F, Andrade EIG. Financiamento, descentralização e regionalização: transferências federais e as redes de atenção em Minas Gerais, Brasil. Ciênc Saúde Colet. [Internet]. abr 2017 [citado em 12 ago 2020]; 22(4):1245-56. Disponível em: http://www.scielo.br/scielo.php?script= sci_arttext\&pid=S1413- 81232017002401245\&lng=pt. doi: http s://doi.org/10.1590/141381232017224.28252016

10. Andrade MC, Castanheira ERL. Cooperação e apoio técnico entre estado e municípios: a experiência do Programa Articuladores da Atenção Básica em São Paulo. Saúde Soc. [Internet]. 2011 [citado em 03 out 2016]; 20(4):980-90. Disponível em: http://www.scielo.br/scielo.php?script= sci_arttext\&pid=S0104-

12902011000400015

11. Silva MAA, Rodrigues VZC. Gestão dos serviços de saúde no Sistema Único de Saúde (SUS) em uma regional de saúde do Distrito Federal (SES-DF): visão do gestor relacionada ao preparo para a função. Rev Eletrônica Gest Saúde [Internet]. 2013 [citado em 10 nov 2016]; 4(3):843-60. Disponível em:

https://periodicos.unb.br/index.php/rgs /article/view/318/305

12. Moreira MR, Ribeiro JM, Ouverney AM. Obstáculos políticos à regionalização do SUS: percepções dos secretários municipais de Saúde com assento nas Comissões Intergestores Bipartites. Ciênc Saúde

Colet. [Internet]. abr 2017[citado em 12 ago 2020]; 22(4):1097-1108.

Disponível em:

http://www.scielo.br/scielo.php?script= sci_arttext\&pid=S1413-

81232017002401097\&lng=en. doi: htt ps://doi.org/10.1590/1413-

81232017224.03742017

13. Celuppi IC, Geremia DS, Ferreira J, Pereira AMM, Souza JB. 30 anos de SUS: relação público-privada e os impasses para o direito universal à saúde. Saúde Debate [Internet]. abr 2019 [citado em 12 ago 2020]; 43(121):302-13. Disponível em: http://www.scielo.br/scielo.php?script= sci_arttext\&pid=S010311042019000200302\&lng=en. doi: https://doi.org/10.1590/01031104201912101 
14. Prado S. A "Federação inconclusa": o papel dos governos estaduais na federação brasileira. In: Rezende F, organizador. $\mathrm{O}$ federalismo brasileiro em seu labirinto: crise e necessidade de reformas. Rio de Janeiro: FGV; 2013. p. 120-197.

15. Ministério da Saúde (Brasil). Portaria $n^{\circ} 2.436$, de 21 de setembro de 2017.

Aprova a Política Nacional de Atenção Básica, estabelecendo a revisão de diretrizes para a organização da Atenção Básica, no âmbito do Sistema Único de Saúde (SUS) [Internet]. Brasília, DF: Ministério da Saúde; 2017 [citado em 11 ago 2020]. Disponível em: https://bvsms.saude.gov.br/bvs/saudele gis/gm/2017/prt2436_22_09_2017.htm 1

16. Beltrammi DGM. Descentralização: o desafio da regionalização para Estados e Municípios. Rev Adm Saúde. 2008 [citado em 25 nov 2015]; 10(41):159163.

17. Mendes Á, Louvison M. O debate da regionalização em tempos de turbulência no SUS [Editorial]. Saúde Soc. [Internet]. 2015 [citado em 25 nov 2015]; 24(2):393-97. Disponível em: http://www.scielo.br/pdf/sausoc/v24n2/ 0104-1290-sausoc-24-02-00393.pdf

18. Moll MF, Goulart MB, Caprio AP, Ventura CAA, Ogoshi AACM. O conhecimento dos enfermeiros sobre as Redes de Atenção à Saúde. Rev Enferm UFPE on line [Internet]. Jan 2017 [citado em 08 fev 2019]; 11(1): 86-93. Disponível em: https://periodicos.ufpe.br/revistas/revist aenfermagem/article/viewFile/11881/1 4338. doi: 10.5205/reuol.9978-884496-1101201711

19. Ministério da Saúde (Brasil). Portaria n 204/GM, de 29 de janeiro de 2007. Regulamenta o financiamento e a transferência dos recursos federais para as ações e os serviços de saúde, na forma de blocos de financiamento, com o respectivo monitoramento e controle [Internet]. Brasília, DF: Ministério da saúde; 2007 [citado em 03 jul 2020]. Disponível em: http://bvsms.saude.gov.br/bvs/saudele gis/gm/2007/prt0204_29_01_2007_co mp.html

20. Ministério da Saúde (Brasil). Portaria $\mathrm{n}^{\circ}$ Portaria $\mathrm{n}^{\circ} 3.992$, de 28 de dezembro de 2017. Altera a Portaria de Consolidação $n^{\circ}$ 6/GM/MS, de 28 de setembro de 2017, para dispor sobre o financiamento e a transferência dos recursos federais para as ações e os serviços públicos de saúde do Sistema Único de Saúde [Internet]. Brasília, DF: Ministério da saúde; 2017 [citado em 03 jul 2020]. Disponível em: http://bvsms.saude.gov.br/bvs/saudeleg is/gm/2017/prt3992_28_12_2017.html

RECEBIDO: 23/04/2019

APROVADO: $27 / 08 / 2020$

PUBLICADO: 06/2021 